\title{
Historias de la Terapia Ocupacional en América Latina: la primera década de creación de los programas de formación profesional ${ }^{1}$
}

Artículo original: Histórias da terapia ocupacional na América Latina: a primeira década de criação dos programas de formação profissional

Autores: Gustavo Artur Monzeli, Rodolfo Morrison, Roseli Esquerdo Lopes

Traducción: Clara Duarte Cuervo ${ }^{2}$ y Gustavo Artur Monzeli ${ }^{3}$

Recibido: 31 de mayo 2021 • Enviado para modificación: 30 de junio 2021 • Aceptado: 6 de julio 2021

Monzeli, G. A., Morrison, R. y Lopes, R. E. (2021). Historias de la Terapia Ocupacional en América Latina: la primera década de creación de los programas de formación profesional (C. Duarte Cuervo y G. A Monzeli, Trad.). Revista Ocupación Humana, 21(2), 113-136. https://doi.org/10.25214/25907816.1134 (Artículo original publicado en 2019).

\section{RESUMEN}

Este artículo tiene como objetivo analizar los procesos históricos que posibilitaron e impulsaron la creación de la Terapia Ocupacional en América Latina. Para delimitar los países estudiados se seleccionaron aquellos que hacen parte de la Confederación Latinoamericana de Terapeutas Ocupacionales - CLATO. Se contactó a los representantes ante esta organización y se les invitó a colaborar en el levantamiento inicial de documentos y en la identificación de personas involucradas en tales procesos en sus países. Respecto a la recolección

\footnotetext{
1 Esta es una traducción original derivada del artículo de investigación "Histórias da terapia ocupacional na América Latina: a primeira década de criação dos programas de formação profissional", escrito en portugués y publicado en el volumen 27 número 2 de 2019 de los Cadernos Brasileiros de Terapia Ocupacional, el cual está disponible en este enlace: https://doi.org/10.4322/2526-8910.ctoAO1631. Como obra derivada, quienes elaboraron esta traducción contaron con el consentimiento de los titulares de los derechos morales del artículo para la elaboración y publicación de esta traducción: Gustavo Artur Monzeli, Roseli Esquerdo Lopes y Rodolfo Morrison Jara. Así mismo, se acogieron a los términos de la licencia Creative Commons Attribution (CC-BY), bajo la cual el artículo original fue publicado.
}

${ }^{2}$ Terapeuta ocupacional. Magíster en Salud Pública. Doctoranda en Terapia Ocupacional, Universidade Federal de São Carlos. Nemocón, Colombia. claradc2002@gmail.com

\section{iD https://orcid.org/0000-0001-6901-7335}

${ }^{3}$ Terapeuta ocupacional. Magíster y doctor en Terapia Ocupacional. Docente, Universidade Federal da Paraíba. João Pessoa, Brasil. gustavo.monzeli@gmail.com https://orcid.org/0000-0002-4147-1840 
y organización de datos, se realizó un recorte histórico de los primeros diez años desde el inicio del primer programa de formación en Terapia Ocupacional en América Latina, esto es, 1956 a 1966. Los países cuyos programas fueron creados en esa década son: Brasil, México, Argentina, Venezuela, Chile y Colombia. Como parte de los resultados, se identificó que las historias de la Terapia Ocupacional y de la creación de los programas de formación en América Latina pasan, entre otros factores, por antecedentes relacionados con las epidemias de poliomielitis y la historia del cuidado en el ámbito del sufrimiento psíquico, además de la presencia de los movimientos de cooperación internacional, la jerarquización de las carreras profesionales y los procesos de subordinación del género femenino en lo que se refiere a la inserción de las mujeres en el mercado de trabajo.

\section{PALABRAS CLAVE}

Terapia Ocupacional, Historia, América Latina

\section{Introducción}

La propuesta de investigación de la cual se deriva este artículo surgió al comprender la necesidad de buscar las distintas interconexiones históricas que condujeron a la llegada de la Terapia Ocupacional a América Latina, así como los movimientos que propiciaron la creación de los primeros programas de formación en los países latinoamericanos. Se trata de una tarea relevante para entender la configuración del campo de la Terapia Ocupacional; también, porque todavía hay un escaso dominio colectivo de las historias de la Terapia Ocupacional en los países de la región.

El objetivo de esta propuesta, entonces, es comprender los procesos históricos de creación de la Terapia Ocupacional, específicamente de la formación profesional, en los países de América Latina. Para lograrlo, el foco está en la identificación de los primeros programas de formación profesional, intentando comprender el contexto socio-político de los países para el momento en el que fueron creados. Adicionalmente, es necesario discutir los procesos de creación de esos primeros programas, las articulaciones que fueron necesarias para dar paso al inicio de la formación profesional y las principales razones que justificaron la creación de estos programas en particular en los países latinoamericanos.

Es importante comprender que cuando evocamos la idea de Terapia Ocupacional nos referimos a diferentes ámbitos, desde las prácticas de atención y cuidado a poblaciones específicas; las propuestas de formación técnica, profesional y académica de un área específica de conocimiento; hasta los procesos de reglamentación e institucionalización de la profesión. Este trabajo se enfoca en la dimensión de los programas de formación profesional. El interés aquí es discutir un análisis de los procesos históricos y de las razones que se usaron para justificar la generación de propuestas de formación profesional por parte de instituciones de educación o de asistencia en los países de América Latina. 
De esta manera, para comenzar se discute brevemente la "idea" de América Latina y se presenta un recorrido histórico de la creación de la profesión en Estados Unidos; posteriormente se contextualizan y analizan los datos generados en esta investigación.

\section{América Latina}

Comenzar con una definición de América Latina, además de importante, es bastante complicado $y$, en algunos momentos, paradójico. Es importante porque responde a la necesidad de localizar histórica y geográficamente una región, algunos idiomas y muchos pueblos distintos y diversos. Complicado, justamente por esa enorme diversidad, no solo de pueblos y culturas, también de discursos sobre esas diversidades. Paradójico, porque para hablar de esa complejidad, con frecuencia se adoptan perspectivas instaladas por las naciones invasoras o colonizadoras, que crean una identidad latinoamericana en oposición y asimetría frente a tales naciones.

El concepto de América Latina es de origen francés, se deriva de la expresión Amerique latine, que fue utilizada por los intelectuales franceses para denominar el imperialismo en México, bajo el dominio de Napoleón III, en la década de 1860 (Bethell, 2009). Sin embargo, de acuerdo con Bethell (2009), existen tres autores que previamente, en diferentes ocasiones, en 1856, hicieron uso del término "América Latina" por primera vez: José María Torres Caicedo, periodista, poeta y crítico colombiano; Francisco Bilbao, intelectual socialista chileno, y Justo Arosemena, jurista, político, sociólogo y diplomático colombo-panameño.
Algunos autores, como Furtado (2007), identifican 20 países en esa región: Argentina, Bolivia, Brasil, Chile, Colombia, Costa Rica, Cuba, Ecuador, El Salvador, Guatemala, Haití, Honduras, México, Nicaragua, Panamá, Paraguay, Perú, República Dominicana, Uruguay y Venezuela. Adicionalmente, los clasifican en Andinos, Atlánticos y Caribeños. Otros autores, como Araújo (2006) y Souza (2011), comprenden la región latinoamericana como todos aquellos países ubicados por debajo del Rio Bravo, entre Estados Unidos y México, es decir, 32 naciones que no hacen parte de la América anglosajona, estos incluyen, además de los 20 previamente citados, a Guyana, Surinam, Belice, Antigua y Barbuda, Aruba, Bahamas, Barbados, Dominica, Granada, Jamaica, Santa Lucía y Trinidad y Tobago.

En esta región se habla predominantemente español, portugués y francés; esto como resultado de la invasión y la colonización. Además, existen centenas de otras lenguas indígenas ya extintas o que están en proceso de extinción por cuenta de los continuos procesos de violencia que han enfrentado estos pueblos, entre ellas, guaraní, tupíes, aimara, quechua, náhuatl, maya y mapuche; incluso, se emplean otras lenguas de origen africano que llegaron mediante el tráfico de personas esclavizadas.

América Latina se configura como región a partir de diversos abordajes. Algunos de estos la comprenden y conceptualizan como "región natural", esto es, "Entendida como una parte de la superficie de la tierra, medida de acuerdo con escalas territoriales diversificadas y caracterizada por la uniformidad resul- 
tante de la combinación o la integración en áreas de elementos de la naturaleza" (Correa, 2003, p. 23, traducción nuestra).

Otros abordajes consideran que la región está determinada por técnicas estadísticas y de medición (Dias, 2009), como lo define Correa (2003): “Un conjunto de lugares donde las diferencias internas entre esos lugares son menores que las existentes entre estos y cualquier elemento de otro conjunto de lugares (...) definir región pasa a ser un problema de medición eficiente y de estadísti-

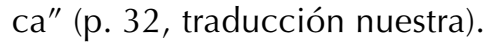

A mediados del siglo XX algunos autores comienzan a construir el concepto de región, reforzando su existencia bajo el capitalismo (Dias, 2009). En este sentido, Correa (2003) explica que "Es en el modo de producción capitalista que el proceso de regionalización se acentúa, marcado por la simultaneidad de los procesos de diferenciación e integración, verificada dentro de la progresiva mundialización de la economía a partir del siglo XV" (p. 44, traducción nuestra).

En esta interpretación de América Latina, "Al rescatar la historia colonial, el papel de los países latinoamericanos insertados en la División Internacional del Trabajo y relacionar explotadores y explotados, estamos acentuando el carácter ideológico de América Latina como región" (Dias, 2009, p. 25, traducción nuestra).

A las definiciones de región, Gomes (1995) agrega que las principales características serían la conciencia regional, el sentimiento de pertenencia y las mentalidades regionales como elementos que revalorizan la dimensión regional como espacio vivido.
Por lo tanto, hablar de una región latinoamericana implica comprender que ese discurso se inserta en una trama compleja de significados y perspectivas, en ocasiones próximos y coherentes, pero en otras opuestos y contradictorios.

\section{Surgimiento de la Terapia Ocupacional en Estados Unidos de América}

Un punto en común en las diferentes narrativas en torno a la historia del surgimiento de la Terapia Ocupacional en Estados Unidos de América son las intervenciones llevadas a cabo por mujeres con diferentes formaciones profesionales, bajo la tutela de hombres que, en general, son los autores de esas narrativas.

Morrison (2016) propone buscar visiones "alternativas" a la historia "oficial" de la Terapia Ocupacional, haciendo énfasis en el lugar central de las mujeres en su desarrollo. Reorganizar la historia de los orígenes de la Terapia Ocupacional podría ser una manera de enfatizar el protagonismo de mujeres en ese proceso, el cual estaría subsumido en situaciones de subordinación del género femenino en diversos contextos históricos.

Es importante comprender que los orígenes de la profesión están íntimamente ligados con la posibilidad de inserción de las mujeres -principalmente blancas y de élites urbanas- en esferas de la vida social que trascienden el espacio doméstico. Se destaca la participación en las instituciones de enseñanza, las instituciones de caridad y beneficencia -especialmente de carácter religioso-, las universidades y también, en parte, como respuesta a demandas del mercado de trabajo (Lopes, 1991; Morrison, 2016), aun cuando la inser- 
ción en esos espacios no ha ocurrido de forma igualitaria a la de los hombres.

Algunos de esos cambios fueron posibles debido al contexto social estadounidense de finales del siglo XIX e inicios del siglo $X X$, en una sociedad marcada por el proceso de transformación del monopolio del capital (Braverman, 1987). En este contexto de transformaciones, algunas mujeres rechazaron el matrimonio y dedicaron sus vidas a la enseñanza, a la enfermería o al trabajo social. Otras, aun estando casadas, pertenecieron a legiones y grupos de caridad o de otro tipo de ayuda social. Esa ampliación de la circulación y de la inserción en diferentes espacios sociales pudo haber forjado referentes femeninos en otras esferas públicas (Morrison, 2016).

La creación de la Hull House en el contexto de la Escuela de Cívica y Filantropía de Chicago hace parte de ese proceso en el cual muchas mujeres jóvenes, estimuladas principalmente por los movimientos feministas de la época (Addams, 1912), transformaron las nociones de ayuda y compromiso social voluntario (basado en perspectivas morales y religiosas) hacia la valoración de sus actividades laborales; reconfiguraron el mercado de trabajo emergente y demandante, y propiciaron su inserción en empleos formales (Morrison, 2016).
La Hull House era un refugio de extrema importancia para el movimiento de mujeres en Chicago (Quiroga, 1995), tanto por su contribución a la formación política, profesional y laboral de muchas mujeres, como por ofrecer apoyo a un número importante de familias, principalmente de inmigrantes (Addams, 2002).

Si desde el androcentrismo la historia de la Terapia Ocupacional se habría iniciado con el tratamiento moral ${ }^{\mathbf{4}}$, desde una perspectiva feminista podría decirse que fue el movimiento de esos refugios sociales el que permitió que muchas mujeres -blancas y de élites urbanas- se inspiraran y comenzaran a desarrollar actividades laborales (Morrison, 2014), ya que las mujeres pobres, las mujeres negras -o pobres y negras- $y$ las mujeres pobres e inmigrantes siempre trabajaron, de una u otra manera, fuera de sus espacios domésticos. Para Morrison (2014), por primera vez aquellas mujeres comenzaron a vislumbrar alternativas de participación social y pudieron optar por distanciarse de las formas tradicionales, como el matrimonio y la familia.

Las mujeres fundadoras de la Hull House buscaban comprender las condiciones y las circunstancias en las que vivían otras mujeres y las familias de inmigrantes, lo que se traducía, por un lado,

\footnotetext{
${ }^{4}$ En el siglo XIX, Philippe Pinel en su trabajo titulado Tratado médico-filosófico sobre la alienación mental comprendía que la locura era un exceso y un desvío por corregir a través del cambio de costumbres y de inculcar nuevos hábitos y comportamientos, sentando las bases de lo que se denominó tratamiento moral. Según Millani y Valente (2008), el tratamiento moral incluía toda la organización y la producción de la rutina asilar, es decir, la sistematización del uso del tiempo y del espacio de los internos, con miras a someter al interno, alejándolo de su mundo para encuadrarlo en un sistema de preceptos médicos y valores morales. Adicionalmente, Magalhães (1989) destaca que "Articulando un discurso que es al mismo tiempo terapéutico y policial, el tratamiento moral funda una ética (o por lo menos rescata para el dominio médico) que se basaba, sobre todo, en el valor de la disciplina y su principal producto social: el trabajo alienado" (p.55-56, traducción nuestra).
} 
en ayuda inmediata y, por otro, en elaboración teórica sobre esos temas, tarea que compartían con la University of Chicago (Metaxas, 2000; Miranda, 2007).

Jane Addams y Ellen Gates Starr, como principales fundadoras de la Hull House a finales de la década de 1880, Julia Lathrop, una de las primeras en incorporarse a esa institución, y Eleanor Clarke Slagle fueron las primeras mujeres responsables de las acciones de la Hull House. Este trabajo en el ámbito de la acción social, de lidiar bajo un enfoque político con demandas sociales de la sociedad capitalista que se levantaba sobre los cimientos estadounidenses, comienza también a ofrecer a algunas de estas mujeres parámetros para acciones que más tarde se convertirían en propuestas en torno a la Terapia Ocupacional (Morrison, 2016).

Adolf Meyer se une a esas mujeres y comienzan a trabajar en reformar el tratamiento que se ofrecía a las personas consideradas como enfermas mentales, teniendo en cuenta que uno de sus presupuestos era que las deplorables condiciones de vida de los pobres y los trabajadores en las ciudades -que crecían a pasos agigantados- podían hacer parte de las causas que favorecían o mantenían situaciones de enfermedad. Adicionalmente, intentaban responder también a las críticas de la época sobre la ausencia de "ciencia" en esos "nuevos métodos" empleados en psiquiatría, publicando algunos artículos generados a partir de esas prácticas (Addams, 1935; Pollard, et al., 2009; Morrison, 2016).

En 1910, Adolf Meyer solicita a Julia Lathrop que le recomiende a una trabajadora social que se pudiera vincular al Hospital Johns Hopkins (en la ciudad de Baltimore), dirigido por él, para tra- bajar con personas consideradas enfermas mentales. Lathrop sugiere a Slagle, quien acepta la tarea. Después de dos años de trabajo, Meyer destacaba a Slagle como la principal referente del servicio de Terapia Ocupacional. Al final de esa experiencia, Slagle regresa a Chicago en 1913 para fundar la escuela de Terapia Ocupacional (Morrison, 2016).

De acuerdo con Morrison (2016), apoyado en Quiroga (1995), las principales dificultades en el desarrollo de la profesión para las primeras terapeutas ocupacionales se centraron en dos aspectos: primero, el posicionamiento dentro del campo de la medicina; y segundo, el hecho de que la Terapia Ocupacional fuera considerada como una profesión de mujeres.

Desde sus inicios la Terapia Ocupacional fue considerada una nueva profesión para mujeres (Lopes, 1991; Metaxas, 2000; Lopes y Hahn, 2004), y para legitimarse como disciplina profesional reconocida se buscaron articulaciones con la medicina, que hasta entonces era un campo masculino $y$, como hoy, con un elevado reconocimiento y poder social. El papel de los hombres dentro del mundo visible de la medicina y el de las mujeres dentro de la invisibilidad de las redes de caridad (Quiroga, 1995), de jóvenes buenas y señoras de bien (Lopes, 1991) hicieron parte de los mecanismos empleados por la primera generación de terapeutas ocupacionales (Lopes, 1991; Morrison, 2016).

Las primeras mujeres consideradas terapeutas ocupacionales fueron Eleanor Clarke Slagle, trabajadora social que se unió a la Hull House; Susan Cox Jonhson, enfermera que pretendía probar que las ocupaciones podrían mejorar 
la salud física y mental de los pacientes internados en contextos hospitalarios, y Susan Elizabeth Tracy, enfermera, una de las pioneras en el uso de las ocupaciones como tratamiento y una de las primeras en sistematizar sus reflexiones (Morrison, 2016; Reis, 2017).

La Primera Guerra Mundial empezó en 1914, sin embargo, Estados Unidos solo se involucró oficialmente en 1917; así, la profesión reconocida en ese mismo año en ese país no surgió como resultado inmediato de la Guerra, pero sí en ese contexto. Tal momento histórico hizo posible un gran desarrollo y expansión de la profesión, ya que la Terapia Ocupacional fue una de las profesiones que hicieron parte de las "auxiliares de reconstrucción", lidiando con lesiones y discapacidades generadas por la Guerra, y participando activamente en los procesos de rehabilitación (Sanz Valer et al., 2013; Morrison, 2016).

La Terapia Ocupacional se expandió en Estados Unidos en las primeras décadas del siglo XX y retomó de manera importante esa expansión durante la Segunda Guerra Mundial; como parte de esa segunda ola de crecimiento, Ilegó a América Latina como programa de formación profesional a partir de la década de 1950.

\section{Método}

Los datos aquí presentados y discutidos fueron obtenidos en dos momentos principales: primero, la identificación de los colaboradores de la investigación; y segundo, la búsqueda de materiales didácticos, registros históricos y producciones científicas sobre la historia de la Terapia Ocupacional en cada país de América Latina.
Para la identificación de los colaboradores se seleccionaron los países miembros de la Confederación Latinoamericana de Terapeutas Ocupacionales - CLATO. Se contactaron sus representantes ante esa organización y se les invitó a colaborar de manera que fuese posible un levantamiento inicial de documentos y sujetos involucrados con tales procesos en los diferentes países.

La CLATO, fundada en 1997 (Gómez Lillo, 2012), cuenta actualmente con los siguientes países miembros: Argentina, Bolivia, Brasil, Chile, Colombia, Costa Rica, El Salvador, Ecuador, México, Panamá, Paraguay, Perú, Uruguay, Venezuela y Puerto Rico (considerado territorio de Estados Unidos de América y no un país independiente).

Por partir de ese grupo, fueron invitados a participar de la investigación todos los delegados de la CLATO (gestión 20152017). La invitación se envió por correo electrónico y los delegados que aceptaron participar respondieron un cuestionario que incluía sus datos de identificación personal y profesional y solicitaba sugerencias de documentos, textos, artículos o materiales didácticos sobre la historia de la Terapia Ocupacional en su país. Los delegados que colaboraron en la investigación en ese primer momento representaban a los siguientes países: Argentina, Brasil, Chile y Colombia.

Dado que el número de respuestas fue bajo, aún después de varios intentos, se inició la búsqueda de los contactos sugeridos por personas que eran o habían sido representantes de Brasil ante la CLATO, a saber: Ricardo Lopes (delegado 20132015), Rosibeth Palm (exdelegada que participó en la gestión de la CLATO entre 2000 y 2011) y Luciana Wertheimer (pre- 
sidenta de la CLATO 2013-2015). De esta manera fueron contactados los representantes de Costa Rica, México, Panamá, Perú, Uruguay y Venezuela.

También, durante el XII Congreso de la CLATO, en octubre de 2017, en México, se logró contactar a los representantes de países que se habían unido recientemente a la CLATO: Bolivia, El Salvador y Paraguay.

Para esos 14 países se buscaron las fechas de inicio de los primeros programas de formación en Terapia Ocupacional, además se identificaron los motivos de creación y las articulaciones nacionales e internacionales que se dieron para dar inicio a la formación en Terapia Ocupacional en América Latina.

Se recibieron recomendaciones de libros, artículos, entrevistas y tesis de maestría y doctorado que abordan, directa o indirectamente, la historia de la Terapia Ocupacional en cada uno de los países. Algunos países como Argentina, Brasil y Colombia cuentan con grupos de investigación específicos sobre la historia de la profesión, lo que facilitó la identificación de los materiales. Otros países como Chile, México y Venezuela tienen producciones importantes, como libros y disertaciones, aunque más dispersas.

Para el análisis y la presentación de los datos recolectados se realizó una delimitación histórica de los primeros diez años desde la creación del primer programa de formación en Terapia Ocupacional en América Latina, es decir, entre los años de 1956 y 1966. Esta decisión se justifica por el interés de identificar los programas pioneros en la formación, fundados en la primera década de desarrollo institucional de la Terapia Ocupacional en los países latinoamericanos. Los países que crearon cursos en ese período fueron Brasil, México, Argentina, Venezuela, Chile y Colombia.

Los textos recolectados y analizados fueron: Brunetto (1975), Magalhães (1989), Lopes (1991, 1997), Nascimento (1991), Soares (1991), Trujillo (2002), Lopes y Hahn (2004), Testa y Spampinato (2010), Gómez Lillo (2012), Testa (2012), Escobar y Sepúlveda (2013), Nabergoi et al. (2013), Rivas et al. (2013), Melo (2015), Bottinelli et al. (2016), Duarte et al. (2016), Morrison et al. (2016), Rodríguez et al. (2016), Cascajares y Ramíres (2017), y Reis (2017).

\section{Resultados y Discusión}

No es posible hablar de una única historia de la Terapia Ocupacional en América Latina; eso sería un equívoco. En primera instancia, porque cuando se decide contar una historia, aun cuando sea claro desde qué punto de vista se hace o considerando cuáles hechos, se dejan de lado otros enfoques y hechos relevantes que no se cuentan o no son traídos al relato (Gigante, 2008).

La historia siempre es contada partiendo de otro momento histórico y desde puntos de vista diversos; solo con esta observación ya podemos comprender que las realidades son diferentes, las épocas distintas y las palabras y sus significados, a su vez, no necesariamente son los mismos. Por eso, cuando elegimos palabras para contar una historia, no solo describimos una cierta realidad, sino que creamos y recreamos esa realidad.

Para Certeau (1982), al buscar el sentido histórico de un acontecimiento 
se encuentran métodos, ideas o incluso maneras de comprenderlo, es decir, existe una confrontación entre pasado y presente, entre aquello que organizó una determinada realidad y aquello que hoy permite pensarla. En esta misma línea, Le Goff (2005) destaca que "No hay realidad histórica acabada que se entregue por sí misma al historiador" (p. 41, traducción nuestra).

En consecuencia, los datos aquí presentados son interpretaciones, relecturas, rearticulaciones y recreaciones de determinadas historias que hablan sobre los movimientos de creación de la profesión de Terapia Ocupacional en diferentes países de América Latina.

\section{Antecedentes y encuadres de la implantación de la Terapia Ocupacional en América Latina}

Las epidemias de poliomielitis y la historia de la locura - principalmente a partir de las ideas del tratamiento moral -, son algunos de los marcos importantes para abordar las historias de la Terapia Ocupacional en los países latinoamericanos.

La poliomielitis y los centros de rehabilitación

La poliomielitis fue durante siglos una causa importante de discapacidad y muerte infantil, hasta la creación de la vacuna contra esta enfermedad a mediados de la década de 1950 (Testa, 2012). En América Latina, los primeros casos de poliomielitis fueron identificados y registrados a finales del siglo XIX en México, en 1906 en Argentina, en 1909 en Chile, en 1911 en Brasil, en 1915 en Colombia y en 1928 en Venezuela. Entre tanto, su reconocimiento como epidemia en esos países ocurrió entre los años 1930 y 1950
(Echezuría, 1974; Malagón y Ávila, 1982; Campos et al., 2003; Laval, 2007; Nascimento, 2010; Testa, 2012; Bottinelli et al., 2016; Cascajares y Ramíres, 2017).

La epidemia de poliomielitis y sus consecuencias fueron uno de los principales argumentos para la creación o la ampliación de los centros de rehabilitación física y los hospitales infantiles, entre otras instituciones de asistencia en el contexto latinoamericano. Lo anterior puede evidenciarse en la creación del Hospital Infantil de México, en 1943 (Cascajares y Ramíres, 2017); el Centro de Rehabilitación Infantil de Chile, en 1947 (Escobar y Sepúlveda, 2013); la Associação Brasileira Beneficente de Reabilitação, en 1954 (Reis, 2017; Reis y Lopes, 2018), entre otros centros de rehabilitación y asociaciones. Estas instituciones tuvieron un crecimiento importante derivado de inversiones nacionales y articulaciones internacionales, y fruto de las preocupaciones generadas por la circulación de personas y mercancías durante el avance de la epidemia (Mendes, 1996). Adicionalmente, hacen parte de las principales instituciones en las que, en la década de 1950, se dio origen a los programas de formación en Terapia Ocupacional.

Este contexto regional en el que los países latinoamericanos se vieron forzados a lidiar con las causas de las epidemias de poliomielitis, al tiempo que se producían técnicas de prevención de la infección y tecnologías de atención a las personas afectadas por la enfermedad, desencadenó el desarrollo de disciplinas relacionadas con la rehabilitación. A su vez, promovió la importación de conocimientos y prácticas de países del norte que, dado el interés económico agroexportador, generaban lazos importantes con países de la región, ya habían pasado 
por esa situación durante o después de la Segunda Guerra Mundial y contaban con mayor información, principalmente en el área de la salud (Bottinelli et al., 2016; Soares, 1991; Sbriller, 1997).

Este proceso desencadenó la creación y expansión de carreras comprendidas hasta entonces como tecnologías médicas, entre ellas la Terapia Ocupacional (Soares, 1991; Gómez Lillo, 2012; Testa, 2012; Rivas et al., 2013; Cascajares y Ramíres, 2017).

Las ocupaciones y el trabajo en el proceso de rehabilitación

Para las historias de la Terapia Ocupacional en los países de América Latina, tan importantes como los centros de rehabilitación física creados o impulsados por los intereses creados en torno a las epidemias de poliomielitis, fueron los hospitales psiquiátricos y las instituciones que lidiaban con las personas tildadas de "locas" o que vivían problemáticas derivadas de cuestiones sociales (Lopes, 2016), como las personas privadas de la libertad, en situación de calle o trabajadoras sexuales.

En Brasil, en el Hospital Dom Pedro $I I$, fundado en 1852 en Rio de Janeiro, las ocupaciones eran objeto de los talleres de sastrería, ebanistería, zapatería, flores, hilado y tejidos, que se basaban de manera importante en una perspectiva del tratamiento moral (Nascimento, 1991; Soares, 1991). En São Paulo, en el Hospital do Juqueri, inaugurado en 1898, los principales usos de la ocupación fueron bajo la forma de praxiterapia o tratamiento a través del trabajo, también, bastante influenciado por el ideario del tratamiento moral (Nascimento, 1991; Soares, 1991).
Esto trae a la escena el uso de actividades, ocupación o trabajo, independientemente de lo que vino a ser denominado como Terapia Ocupacional y antes de la creación de sus programas de formación profesional, como una forma de "cuidado" o "tratamiento" del sufrimiento psíquico institucionalizado. Al mismo tiempo, interesaba a la institución en tanto ayudaba en el control de los cuerpos, al mantenimiento del orden y en tareas básicas para su funcionamiento, como trabajo no remunerado y muchas veces obligatorio (Nascimento, 1991).

En 1946, Nise da Silveira fundó el Serviço de Terapêutica Ocupacional do Centro Psiquiátrico Nacional, en el Engenho de Dentro - Rio de Janeiro, y puso en el centro de su atención el potencial expresivo de las actividades escogidas libremente para el proceso de comprensión de la "locura" y ayuda a los pacientes, sin que estas necesariamente fueran útiles para el hospital, aunque también podían serlo (Brunetto, 1975; Magalhães, 1989; Nascimento, 1991;).

En Colombia, a mediados del siglo XIX, la ocupación/trabajo comienza a ser utilizada como un medio para impedir o salir de la "vagancia" y ascender a la moral católica, lo que también se vinculaba con una perspectiva económica y de "progreso", necesarios para la consolidación de la república (Duarte et al., 2016). La alusión al trabajo con la población pobre, con los reclusos en los presidios y con las prostitutas hacía parte de esa concepción de que era posible rehabilitar "problemáticas sociales" a través de la ocupación o del trabajo (Duarte et al., 2016). La enseñanza y la práctica de oficios seguían funcionando como estrategias para combatir las malas costumbres 
y la pobreza, bajo los preceptos de la moral cristiana y principalmente católica (Duarte et al., 2016).

En Argentina, a finales del siglo XIX, el tratamiento moral era utilizado en el Hospicio de las Mercedes, donde se ofrecían diferentes talleres, dependiendo de las posibilidades físicas y psíquicas de los internos; la salud o ausencia de enfermedades se pensaba en términos de productividad y, por lo tanto, la inserción socio-laboral era de suma importancia (Gómez, 2007).

En Chile, alrededor de la década de 1930, existían publicaciones de psiquiatras que defendían el tratamiento moral y la denominada laborterapia, en los que se utilizaban los talleres y las ocupaciones para promover beneficios dentro de lo que se entendía como tratamiento y generar mayor independencia a los pacientes (Gómez Lillo, 2012).

Así, se puede percibir una articulación histórica alrededor de los discursos previos a los orígenes de la Terapia Ocupacional en los países latinoamericanos, transitando entre la creación de una tecnología de atención con fines de rehabilitación física, pensada como respuesta a las epidemias en la región, y la justificación técnica y científica de un trabajo que ya ocurría en hospitales psiquiátricos, con el propósito de dar forma profesional a técnicas cuyo uso articulaba, en mayor o menor grado, presupuestos del tratamiento moral.

\section{Cooperación internacional}

Debido a la historia de las epidemias de poliomielitis, diferentes proyectos de cooperación internacional tuvieron un papel importante en la creación y el desarrollo de algunos de los primeros programas de formación profesional en Terapia Ocupacional en el contexto latinoamericano. Entre las agencias y organizaciones internacionales citadas como importantes para la referida creación se destacan la Organización de las Naciones Unidas -ONU, la Organización Internacional del Trabajo -OIT, la Organización Mundial de la Salud -OMS y la Organización Panamericana de la Salud -OPS.

Al tener parte de sus orígenes en organismos creados tras el fin de la Primera Guerra Mundial y compartiendo el contexto histórico derivado de la Segunda Guerra Mundial -que creó muros entre el mundo capitalista y el socialista, envueltos en la Guerra Fría-, esas organizaciones se volcaron a una Cooperación Internacional para el Desarrollo que comienza a ser institucionalizada con la emergencia de normas, discursos, prácticas, agendas y comportamientos de actores definidos de manera más organizada, regular y previsible (Milani, 2014).

Los principales proyectos de cooperación internacional que surgieron y se diseminaron en el siglo XX tienen una conexión importante con el fin de la Primera Guerra Mundial y con la efervescencia de la Revolución Socialista originada en Rusia. En ese período se creía que los regímenes elegidos democráticamente no tomarían la decisión de ir a la guerra, excepto en situaciones de defensa propia, y que solamente los regímenes que no se elegían de forma democrática tendrían la tendencia a generar conflictos bélicos. Así, una de las estrategias para evitar nuevos conflictos consistió en extender por el mundo las democracias liberales (Llistar, 2009). 
Al tiempo que algunos acuerdos internacionales comenzaban a ganar centralidad en las discusiones globales y con el inicio de la Segunda Guerra Mundial, esta perspectiva liberal fue cuestionada, toda vez que la Alemania nazi y la Italia fascista habían sido creadas por líderes elegidos popularmente, o sea, la idea de que la diseminación de las democracias pondría fin a las guerras luego fue rechazada.

En la década de 1960, en el contexto de la Guerra Fría, con la crisis de los misiles en Cuba, la carrera espacial y las intervenciones militares de Estados Unidos de América y la Unión de Repúblicas Socialistas Soviéticas, la importancia militar y diplomática comenzó a ser relativizada en detrimento de la emergencia de otros actores, como empresas multinacionales y organismos internacionales como la ONU o la Comunidad Europea (Llistar, 2009).

Las razones iniciales para la institucionalización de estrategias de cooperación y desarrollo no pueden disociarse de la Guerra Fría, dado que las rivalidades este-oeste, a partir de 1945, fueron un factor determinante para que, en el campo liberal occidental, se tomaran decisiones en el sentido de promover la cooperación entre las naciones del "mundo libre" (Milani, 2014). Se construye una percepción de que las relaciones internacionales y de cooperación (y regulación) son realizadas por actores que no se restringen a los estados-nación, en una red de interdependencia compleja (Keohane y Nye, 1977).

Algunos autores, como Llistar (2009), analizan ese sistema mundial a partir de la constatación de que tales interferencias internacionales comienzan a configurarse como semipermeables, esto quiere decir, permeables a algunos fenómenos como la relación con el capital, las ofensivas militares etc.; pero, al mismo tiempo, impermeables a otros fenómenos como la circulación de personas de países "pobres" y periféricos hacia países "ricos" y centrales en la economía mundial. "Los poros selectivos de las fronteras de los Estados hacen que seamos más interdependientes, aunque interdependencia para nada signifique simetría" (Llistar, 2009, p. 20).

En la lógica de las discusiones sobre centros globales y periferias económicas es importante comprender que el "subdesarrollo" es resultado de relaciones históricas. Es decir, ninguna antigua colonia africana, asiática o latinoamericana puede explicar su presente sin reiterar que su historia ha sido atravesada por largos años de invasiones, exterminios, espoliaciones, tráfico de personas esclavizadas, racismos etc. Al mismo tiempo, la industrialización y la prosperidad económica no se deben solo a las habilidades y las circunstancias de los países que se consideran "desarrollados", sino, igualmente, a la sobreexplotación humana y física de África, Asia y América Latina (Llistar, 2009).

Esas "relaciones internacionales" también pueden ser conceptualizadas como: "Interferencias transnacionales que se producen entre grupos sociales locales o transnacionales que habitan distintos países o entre sus Estados (Llistar, 2009, p. 28), donde el término "relaciones" no coincide con la realidad, puesto que no demuestra direccionalidad, aunque se presenta de forma neutra. En contrapartida, "interferencia", por ser vectorial, consigue reflejar mejor esos procesos internacionales, indicando las asimetrías (Llistar, 2009). Es interesante percibir que 
tales interferencias no son necesariamente negativas, pues existen, por ejemplo, interferencias positivas como las tecnologías de bajo costo, fármacos, sistemas de telefonía e internet etc.

El concepto de "desarrollo" empieza a ser utilizado después de la Segunda Guerra Mundial. La descolonización e independencia de los países considerados del Tercer Mundo (los que no eran considerados países capitalistas desarrollados ni países socialistas) condujo a un cuestionamiento sobre el origen de las desigualdades entre los países, la noción de desarrollo se equiparó a la de crecimiento económico y a esta se le atribuyó un doble objetivo: modernizar y reproducir paulatinamente el modelo establecido por el Primer Mundo. Desde este punto de vista el problema del subdesarrollo recaía exclusivamente en el sur, y de su responsabilidad dependía el crecimiento, la modernización y la industrialización. Y la única cosa que el norte podría hacer sería ofrecer ayuda, una ayuda que fuese concebida como intrínsecamente buena, necesaria y humanitaria (Llistar, 2009).

En la práctica, el "deseo de desarroIlo" contribuyó a las relaciones de cooperación internacional, que tuvo como uno de sus aportes la asesoría técnica de especialistas y agencias de cooperación de países del norte a países del sur global (Llistar, 2009). Dentro de esta lógica, pactos militares, apoyo a dictaduras y programas de desarrollo estuvieron íntimamente ligados en América Latina (Escobar, 1998).

Con estos procesos de cooperación internacional, América Latina comenzó a recibir asesoría de consultores de la ONU, vía articulación con la OIT y la OMS, para la realización de cursos de capacitación, auxilio en el proceso de implementación de programas de rehabilitación, bien para accidentes de trabajo o por la poliomielitis, dentro de otros auxilios técnicos.

A finales de la década de 1940, la ONU pasó a asumir la coordinación, la planeación y la provisión de refuerzos en áreas orientadas a la rehabilitación, partiendo de diversas organizaciones. La OMS se hizo responsable de la formación de profesionales de rehabilitación como médicos, enfermeros, terapeutas ocupacionales, fisioterapeutas, entre otros. La OIT tenía bajo su responsabilidad la rehabilitación profesional, vía seguridad social, y la UNESCO se enfocaba en la educación especial (Soares, 1991).

En 1951, la ONU comienza a enviar emisarios a América Latina con el fin de identificar posibles lugares para la instalación de un centro de rehabilitación. Uno de los escogidos fue el Hospital das Clínicas da Faculdade de Medicina da Universidade de São Paulo (HC-USP), que ya tenía reconocimiento internacional (Soares, 1991).

En Brasil, tanto la Associação Brasileira Beneficente de Reabilitação como el Hospital das Clínicas da Faculdade de Medicina da Universidade de São Paulo (HC-USP) comenzaron a ser asesorados por esas agencias internacionales para introducir prácticas especializadas en rehabilitación, hasta entonces inexistentes en el país (Soares, 1991). Adicionalmente, la creación de los programas de formación profesional también fue uno de sus resultados, es el caso de la Terapia Ocupacional, entre otras profesiones que fueron creadas o impulsadas en ese contexto. 
También en relación con la formación profesional, algunos técnicos y profesionales que actuaban en el área de rehabilitación en el Hospital das Clínicas da Faculdade de Medicina da Universidade de São Paulo (HC-USP) fueron enviados a Estados Unidos para especializarse. Soares (1991) señala que ese fue el caso de Neyde Tosetti Hauck, asistente social y enfermera, quien fue a estudiar Terapia Ocupacional en la University of New York con financiación de la OMS, además de los terapeutas ocupacionales Ilevados a Brasil para contribuir con los programas de formación profesional (Soares, 1991).

En Argentina, la creación de la Escuela Nacional de Terapia Ocupacional -ENTO, en 1959, hizo parte de un acuerdo entre los gobiernos argentino y británico, por medio de un convenio de cooperación técnica con la OMS (Nabergoi et al., 2013). Incluso antes de la creación de la ENTO, al igual que en Brasil, estudiantes y profesionales argentinos comenzaron a recibir becas de estudios para realizar su formación profesional en otros países, así como financiamiento para visitas a servicios especializados, a lo que se sumaba la recepción de especialistas de otros países en las instituciones argentinas (Nabergoi et al., 2013).

Tales acuerdos entre los gobiernos de Argentina e Inglaterra y la OMS garantizaron el cubrimiento de los costos de la formación profesional de estudiantes y profesionales argentinas en la Dorset House, en Oxford. En 1959 tiene lugar el viaje de un equipo docente de esta institución a Buenos Aires, con el fin de organizar y crear el primer programa de formación de terapeutas ocupacionales en Argentina (Nabergoi et al., 2013).
En Venezuela esa creación pionera ocurrió en el Instituto Venezolano de Seguros Sociales, tuvo un apoyo importante de la ONU y resultó de la articulación de tres fisioterapeutas y una terapeuta ocupacional de Canadá para la creación de la Escuela Nacional de Rehabilitación en 1959 (Rivas et al., 2013). Ya el segundo programa de formación en Terapia Ocupacional en ese país, en el año 1967, se derivó de la articulación entre el gobierno venezolano, a través del Programa Nacional de Rehabilitación, la OPS y la OMS, que llevaron como consultora técnica a Carmen Forn, argentina, jefe del Departamento de Terapia Ocupacional del Instituto Nacional de Rehabilitación en Buenos Aires (Rivas et al., 2013).

En Chile, en 1962 se intensificaron los programas de formación profesional para la rehabilitación física. A través de una articulación entre la OPS y el Servicio Nacional de Salud se destinaron fondos importantes para equipar los servicios de rehabilitación en Santiago y otras ciudades de Chile. Al mismo tiempo, se recibían especialistas en rehabilitación para elaborar la propuesta de creación de un centro nacional de rehabilitación (Gómez Lillo, 2012; Escobar y Sepúlveda, 2013).

En el mismo año, 1962, la Clínica Psiquiátrica Universitaria, en Santiago, recibió por tres meses a la terapeuta ocupacional estadounidense Beatrice Wade, a través del Programa de Cooperación Interamericano. Esta terapeuta ocupacional organizó algunos cursos y también un departamento de Terapia Ocupacional en esa clínica (Gómez Lillo, 2012; Escobar y Sepúlveda, 2013).

También durante los inicios de la década de 1960, en Chile fueron creados a 
través del Ministerio de Salud los convenios Chile 21 y Chile 5.000 entre la OPS y el gobierno chileno, los cuales permitieron, entre otras cosas, la creación de centros de medicina física y rehabilitación y la llegada de especialistas para contribuir con programas de formación de terapeutas ocupacionales (Gómez Lillo, 2012).

Uno de los resultados de estas articulaciones internacionales fue la posibilidad de crear el programa de formación en Terapia Ocupacional de la Universidad de Chile; al mismo tiempo, se ofrecían becas a profesionales y estudiantes chilenas para realizar la formación en Terapia Ocupacional en la Escuela Nacional de Terapia Ocupacional de Argentina (Gómez Lillo, 2012). Reis (2017) trae el relato de una brasileña que también recibió esa beca para su formación en Argentina.

Además de las articulaciones internacionales con Estados Unidos, Canadá, Inglaterra y otros países de Europa, es interesante encontrar que, en la década de 1960, el tránsito alrededor de los programas de formación se configuró también entre países latinoamericanos. Otros ejemplos en ese ámbito son el desplazamiento de estudiantes de Honduras y El Salvador al programa de formación de México en 1961, además de una estudiante de Perú y otra de Uruguay en el año 1962 y, en 1964, de una estudiante de Panamá y otra de Costa Rica (Cascajares y Ramíres, 2017). Así mismo, una estudiante de Panamá realizó su formación en el programa venezolano en 1964 (Rivas et al., 2013).

\section{Implantación de los programas de formación}

La década de 1950 marca el inicio de la creación de los programas de formación profesional en Terapia Ocupacional en los países de América Latina. El proceso de creación de esos programas estuvo ligado a factores internos y contextuales de cada país y también a factores internacionales, como ya se ha expuesto.

Es importante destacar que en países como Argentina, Brasil y México, antes de que los programas de formación fueran creados, existieron cursos de capacitación técnica esporádicos y en diferentes formatos $^{5}$. Por lo tanto, la llegada de la profesión en algunos países es anterior a la creación de los programas de formación, sea por convenios internacionales que trajeron terapeutas ocupacionales de otros países o que financiaron la formación de terapeutas ocupacionales latinoamericanas en Estados Unidos o en países de Europa, o incluso, por la formación técnica de personas ya vinculadas a hospitales o centros de rehabilitación para que trabajaran en áreas específicas.

Con los dados obtenidos a través de los documentos recopilados fue posible identificar los primeros programas de formación en Terapia Ocupacional creados en los países latinoamericanos, nueve en el periodo analizado, como se muestra en la tabla 1.

\footnotetext{
${ }^{5}$ Aquí se entienden como "cursos de capacitación", los cursos realizados en Argentina antes de la creación de la ENTO (Bottinelli et al., 2016); en Brasil, por ejemplo, los cursos de Terapia Ocupacional ofrecidos por Nise da Silveira (Soares, 1991), y en México, los cursos de capacitación para enfermeras que ejercerían en ese ámbito (Cascajares y Ramíres, 2017).
} 
Tabla 1. Identificación de programas de formación profesional en Terapia Ocupacional creados en América Latina en los diez primeros años

\begin{tabular}{|c|c|c|c|}
\hline País & Institución & Ciudad & Año de inicio \\
\hline Brasil & $\begin{array}{l}\text { Escola de Reabilitação do Rio de } \\
\text { Janeiro - Associação Brasileira } \\
\text { Beneficente de Reabilitação }\end{array}$ & Rio de Janeiro & 1956 \\
\hline México & Hospital Infantil de México & $\begin{array}{l}\text { Ciudad de } \\
\text { México }\end{array}$ & 1957 \\
\hline Brasil & $\begin{array}{l}\text { Instituto de Reabilitação do } \\
\text { Hospital das Clínicas da } \\
\text { Universidade de São Paulo }\end{array}$ & São Paulo & 1958 \\
\hline Argentina & $\begin{array}{l}\text { Escuela Nacional de Terapia } \\
\text { Ocupacional - Universidad } \\
\text { Nacional de San Martin }\end{array}$ & Buenos Aires & 1959 \\
\hline Venezuela & $\begin{array}{l}\text { Instituto Venezolano de los } \\
\text { Seguros Sociales - Escuela } \\
\text { Nacional de Rehabilitación }\end{array}$ & Caracas & 1959 \\
\hline Brasil & $\begin{array}{l}\text { Instituto Universitário de } \\
\text { Reabilitação da Faculdade de } \\
\text { Medicina do Recife }\end{array}$ & Recife & 1962 \\
\hline Brasil & $\begin{array}{l}\text { Faculdade de Ciências Médicas de } \\
\text { Minas Gerais }\end{array}$ & Belo Horizonte & 1962 \\
\hline Chile & $\begin{array}{l}\text { Facultad de Medicina - } \\
\text { Universidad de Chile }\end{array}$ & Santiago & 1963 \\
\hline Colombia & $\begin{array}{l}\text { Facultad de Medicina - } \\
\text { Universidad Nacional de } \\
\text { Colombia }\end{array}$ & Bogotá & 1966 \\
\hline
\end{tabular}

Fuente: documentos recopilados en la investigación.

El primer programa de formación en Terapia Ocupacional en América Latina fue creado en 1956, en la ciudad de Rio de Janeiro (Brasil), vinculado a la Associação Brasileira Beneficente de Reabilitação (ABBR). Ofrecía una formación de nivel técnico, con dos años de duración; capacitó a siete mujeres en su primera promoción (REIS, 2017).
El segundo programa de formación fue creado en 1957 en la ciudad de México (México), estaba vinculado al Hospital Infantil de México. También era de nivel técnico y tenía una duración de seis meses. En él se capacitaron en Terapia Ocupacional seis enfermeras que ejercían en el mismo hospital. En el mismo año hubo una nueva oferta del curso con una du- 
ración de un año, en esa oportunidad se graduaron otras seis terapeutas ocupacionales (Cascajares y Ramíres, 2017).

El tercer programa en América Latina, creado en 1958 en São Paulo (Brasil), estaba vinculado al Instituto de Reabilitação do Hospital das Clínicas da Universidade de São Paulo. Este curso fue el resultado de una colaboración y articulación de diversas organizaciones como la ONU, la OMS y la OIT. Tenía una duración de dos años y la primera promoción se graduó en 1959, con cuatro terapeutas ocupacionales (Melo, 2015).

Buenos Aires (Argentina) fue sede del cuarto programa, creado en 1959 como Escuela Nacional de Terapia Ocupacional en la Universidad Nacional de San Martin. Este curso tenía una duración de tres años y fue creado siguiendo los lineamientos de la Federación Mundial de Terapeutas Ocupacionales (WFOT) ${ }^{\mathbf{6}}$ (Bottinelli et al., 2016).

En 1959 se creó el quinto programa de formación en Caracas (Venezuela), vinculado al Instituto Venezolano de Seguros Sociales, en la Escuela Nacional de Rehabilitación. Este programa era de nivel técnico, con una duración de tres años y graduó a ocho terapeutas ocupacionales (Rivas et al., 2013).

El sexto programa latinoamericano, creado en 1962, en Recife (Brasil), estuvo vinculado al Instituto Universitário de Reabilitação da Faculdade de Medicina do Recife. Era un curso de capacitación técnica en "rehabilitación", tenía una duración de dos años y los estudiantes optaban durante el curso por la Fisioterapia o la Terapia Ocupacional
(Reis, 2017). En su primera oferta se graduaron 34 profesionales entre fisioterapeutas y terapeutas ocupacionales.

Nuevamente en Brasil, el séptimo programa de formación, creado en 1962 en Belo Horizonte, estuvo vinculado a la Faculdade de Ciências Médicas de Minas Gerais. Allí se crearon cursos técnicos en rehabilitación para Fisioterapia y Terapia Ocupacional (Reis, 2017).

El programa de formación creado en 1963 en Santiago (Chile), vinculado a la Escuela de Tecnología Médica de la Facultad de Medicina de la Universidad de Chile fue el octavo en América Latina. La primera oferta tenía duración de tres años y tres estudiantes participaron de la primera promoción. Así como en Argentina, esa oferta seguía las directrices de la WFOT (Gómez Lillo, 2012).

En 1966 se creó el noveno programa de formación en América Latina, en Bogotá (Colombia), vinculado a la Facultad de Medicina de la Universidad Nacional de Colombia. Se categorizaba como de nivel técnico y tenía duración de tres años, en su primera promoción se graduaron nueve terapeutas ocupacionales (Rodríguez, et al, 2016).

\section{Jerarquización de carreras profesionales y subalternización de género}

En este punto es posible identificar cómo la historia de la creación de la Terapia Ocupacional, de forma general y en especial en América Latina, más allá de los contextos políticos, económicos, sociales y de los procesos de cooperación internacional para atender las epide-

${ }^{6}$ La primera edición de los lineamientos mínimos de formación promulgados por la WFOT es de 1958. 
mias, se relaciona con la organización y creación de técnicas y profesiones en el área de rehabilitación en salud, al mismo tiempo que dialoga con la inserción de las mujeres en la educación técnica superior y en el mercado de trabajo.

En la historia de las instituciones de educación superior, hasta hace muy poco estas no eran un espacio para mujeres ${ }^{7}$, constituyéndose en un ámbito privilegiado de reproducción de las desigualdades de género y de fortalecimiento de la división sexual del trabajo. Los hombres tenían el reconocimiento social por su integración exitosa en la esfera pública, incluso en la educación superior, mientras las mujeres eran invisibilizadas, también en lo educativo, y circunscritas a la esfera privada (Papadópolus y Radakovich, 2006).

Hasta 1950, con excepción de Costa Rica, Cuba, Panamá y Uruguay, el porcentaje de mujeres en las instituciones de educación superior de la mayoría de los países latinoamericanos estaba claramente por debajo de la distribución demográfica de la población total de cada país (Bonder, 1994). Solo hasta finales de la década de 1980, prácticamente en todos los países latinoamericanos, excepto Colombia y Guatemala, las mujeres eran un poco más del $40 \%$ de la población estudiantil en las universidades (Braslavsky, 1994).

En este proceso de aumento de la participación de las mujeres en la educación superior de los países latinoamericanos, la Terapia Ocupacional así como otras profesiones creadas o impulsadas a partir de los años 50 tienen una gran impor- tancia, ya que ayudaron a reconfigurar el papel de las mujeres en la educación superior, en el mundo del trabajo y, en general, en el contexto social.

Así como en Estados Unidos, por ejemplo, la Terapia Ocupacional en sus inicios en América Latina articula dos características principales: en primer lugar, ser una profesión considerada subalterna a la Medicina en el contexto general del área de la salud y, más específicamente, en la rehabilitación; en segunda instancia, ser considerada una profesión para mujeres $y$, justamente por eso, de menor valor en la jerarquía de las profesiones.

Testa y Spampinato (2010) señalan que, en el contexto argentino, se crearon carreras complementarias a la Medicina como forma de contribuir con respuestas a las demandas sociales y de salud frente a las cuales los saberes y profesiones hegemónicas de la época no respondían. Incluida en ese grupo, la Terapia Ocupacional hacía parte de una propuesta de equipo de diferentes profesionales y técnicos coordinados por médicos (Macdonald, 1959; Gonzalez, 1959; Testa y Spampinato, 2010).

Dentro de esta misma perspectiva, la creación de la Terapia Ocupacional en Argentina, según Testa y Spampinato (2010):

Estuvo atravesada por un sistema de género que estableció jerarquías, privilegios y desigualdades; delimitando un campo profesional ligado a un mundo considerado como naturalmente femenino constituido por las actividades de la vida diaria, las actividades arte-

\footnotetext{
${ }^{7}$ En Brasil, por ejemplo, el acceso de las mujeres a la educación superior fue posible solo a finales del siglo XIX (Queiroz, 2000).
} 
sanales y manuales, el cuidado de los niños y una cuota de amor y abnegación para llevar adelante el desafío de la rehabilitación. (p.178)

Con el acceso de mujeres a la educación superior, de modo bastante común y con raras excepciones, esa inserción se dio como una reedición dentro del encuadre de los roles sociales tradicionalmente atribuidos a las mujeres como el cuidado en el ámbito de los procesos de rehabilitación en salud y en la educación especial-, con la consiguiente subordinación a la figura masculina del médico ${ }^{\mathbf{8}}$ y en trabajos que se aproximaban a los valores del voluntariado (Lopes y Hahn, 2004; Nabergoi et al., 2013; Bottinelli et al., 2016; Morrison et al., 2016).

En esa lógica y en el contexto de la Terapia Ocupacional, al tiempo que el acceso a la educación superior y universitaria facilitó la inserción y la participación en el mercado de trabajo y en diferentes espacios sociales para algunas mujeres — hasta entonces alejadas de ese universo-, dicha inserción fue posible mediante el acceso a carreras con menor prestigio académico y, por ello, con perspectivas teóricas y prácticas directamente subordinadas a la visión masculina hegemónica de algunas profesiones.

Si revisamos los primeros programas de formación en Terapia Ocupacional en cada uno de los países aquí analizados, identificaremos que la mayoría de ellos, esto es, los de Brasil, México, Argentina, Venezuela y Colombia, excepto Chile, fueron de nivel técnico. Esto significa que en esos países la Terapia Ocupacional ini- cia su trayectoria a través de programas de formación técnica (en oposición a una formación universitaria), comprendida y vinculada a otras tecnologías médicas y que, por esto, debía justificar sus prácticas en el discurso médico hegemónico, estando sometida a esos conocimientos.

Lo anterior también puede observarse, por ejemplo, al identificar que la dirección de los primeros programas de formación estaba bajo la responsabilidad de médicos, no de terapeutas ocupacionales o de otras profesionales, como se expone a continuación.

En Brasil, en el primer programa de formación, en la Associação Brasileira Beneficente de Reabilitação, el Consejo Técnico (conformado por médicos) era el que orientaba la formación (Reis, 2017).

En México, los primeros programas de formación se iniciaron en el servicio de rehabilitación infantil, bajo la responsabilidad del médico Alfonso Thoen (Cascajares y Ramíres, 2017).

En Venezuela, la dirección del programa estaba ligada a la dirección del Servicio de Rehabilitación, con el médico Alejandro J. Rhode, quien aún contaba con el apoyo de dos terapeutas ocupacionales de la Federación Mundial de Terapeutas Ocupacionales, May Hamilton y Katherine de Brecht (Rivas et al., 2013).

En Chile, el médico Livio Paolinelli estuvo al frente de la coordinación del primer programa de formación, mientras terapeutas ocupacionales ocupaban el cargo de vicecoordinador. La primera terapeuta ocupacional en ocupar la coordi-

\footnotetext{
${ }^{8}$ En la historia de la Medicina, la profesión ha sido ejercida mayoritariamente por hombres. En Brasil, en la década de 1970, las mujeres sumaban 11\% de los profesionales de Medicina (Machado, et al, 2010).
} 
nación del programa fue Margarita León de Pérez-Guerri, en 1963 (Gómez Lillo, 2012; Morrison et al., 2016).

Por su parte en Colombia, la dirección del primer programa estuvo a cargo del director del Departamento de Rehabilitación, el médico Jorge Pardo Ruiz, conjuntamente con las terapeutas ocupacionales Patricia Ann Lang de Pardo, estadounidense graduada en la Western Michigan University, y Alicia Trujillo Rojas, colombiana graduada en la University of New York (Trujillo, 2002; Rodríguez, et al, 2016).

De este modo, el proceso histórico de creación de la Terapia Ocupacional en América Latina incluye, entre otros factores y discursos importantes, esta articulación fundamental entre la creación de una carrera técnica auxiliar a la Medicina, con la posibilidad de inserción de las mujeres en la educación superior de carácter técnico, o sea, de manera subordinada.

\section{Consideraciones finales}

Trabajar con la historia, o mejor, con las diferentes posibilidades y perspectivas para organizar y analizar los procesos históricos es desafiante $y$, al mismo tiempo, extremamente necesario.

En el intento de organizar los discursos y las prácticas de un determinado momento histórico podemos reflexionar sobre sus significados, sus necesidades y también sus posibilidades.

Con lo que hasta aquí se recopiló y con las organizaciones generadas fue posible identificar que las historias de la Terapia Ocupacional y la creación de programas de formación profesional en América Latina tienen antecedentes relacionados con el proceso de expansión de equipos profesionales de rehabilitación, debido a las epidemias de poliomielitis y a la propia historia del "cuidado" de la locura.

Adicionalmente, esos programas de formación fueron impulsados por los movimientos de cooperación internacional, configurando la creación de técnicas y profesiones con menor prestigio en las jerarquías de las carreras médicas, las paramédicas, como eran denominadas, articulando procesos de subordinación del género femenino, en lo que se refiere a la inserción de las mujeres en la educación superior universitaria y, para una parte de ellas, en el mercado de trabajo.

El esquema aquí trazado, que reúne fragmentos de historias de la Terapia Ocupacional en América Latina, tiene características importantes que lo localizan en un tiempo y en un contexto, pero que permiten cuestionamientos y proyecciones, sobre todo en relación con lo que las terapeutas ocupacionales latinoamericanas (y quienes las forman) quieren y pueden producir en su tiempo histórico.

Financiación: este trabajo de traducción se realizó con el apoyo de la Coordenação de Aperfeiçoamento de Pessoal de Nível Superior - Brasil (CAPES) - Código de Financiamiento 001.

Editora responsable: Aida Navas 


\section{Referencias}

Addams, J. (1912). Twenty years at hull house with autobiographical notes. MacMillan.

Addams, J. (1935). My friend Julia Lathrop. MacMillan.

Addams, J. (2002). The long road of woman's memory. University of Illinois Press.

Araujo, M. C. (2006). Prospectos da democracia na América Latina em 2006. CPDOC/FGV.

Bethell, L. (2009). O Brasil e a ideia de "América Latina" em perspectiva histórica. Estudos Históricos, Rio de Janeiro, 22(44), 289-321. https://doi.org/10.1590/S010321862009000200001

Bonder, G. (1994). Mujer y educación en América Latina: hacia la igualdad de oportunidades. Revista Iberoamericana de Educación, 3(6), 9-48. https://doi.org/10.35362/ rie601206

Bottinelli, M. M., Nabergoi, M., Mattei, M. C., Manuel, F. J., Díaz, F. M., Spallato, N. M., Mulholland, M., Martínez, M. M., García, A. M., Briglia, J. y Daneri, S. M. (2016). Reflexiones sobre los orígenes de la formación en Terapia Ocupacional en Argentina. Revista Ocupación Humana, 16(2), 11-25. https://doi.org/10.25214/25907816.129

Braslavsky, C. (1994). Desigualdades educativas de jóvenes y mujeres en América Latina y el Caribe. Unesco.

Braverman, H. (1987). Trabalho e capital monopolista: a degradação do trabalho no século XX. Guanabara.
Brunetto, L. (1975). Terapia ocupacional: correlação teórico-prática. Revista da Associação Brasileira de Psiquiatria, 5(19), 213-226.

Campos, A. L. V., Nascimento, D. R. y Maranhao, E. (2003). A história da poliomielite no Brasil e seu controle por imunização. História das Ciências da Saúde, Manguinhos, 10 (sup. 2), 573-600. https://doi. org/10.1590/S0104-59702003000500007

Cascajares, H. C. D. y Ramíres, G. P. (2017). Terapia ocupacional en México: 60 años transformando vidas. Imagia.

Certeau, M. A. (1982). Escrita da história. Forense Universitária.

Correa, R. L. (2003). Região e organização espacional. Ática.

Dias, W. S. (2009). A ideia de América Latina nos livros didáticos de Geografia [Tesis de Maestría, Universidad de São Paulo]. Biblioteca Digital USP. https://doi. org/10.11606/D.8.2009.tde-11122009$\underline{154104}$

Duarte, C., Fernández, A., Cruz, J. y García, S. (2016). Precursores de la Terapia Ocupacional en Colombia: sujetos, instituciones, oficios. Revista Ocupación Humana, 16(2), 93-109. https://doi. org/10.25214/25907816.140

Echezuría, E. (1974). Estado de la poliomielitis en Venezuela. Bulletin of the Pan American Health Organization, 8(1), 66-71.

Escobar, A. (1988). La invención del Tercer Mundo: construcción y deconstrucción del desarrollo. Norma, 1998. 
Escobar, P. y Sepúlveda, R. (2013). Escuela de Terapia Ocupacional Universidad de Chile: aportes para una historia posible. ETOUCH.

Furtado, C. (2007). A economia latino-americana. Companhia das Letras.

Gigante, M. A. (2008). História oral de idosos asilados em São Carlos - SP: velhice, asilo e memória da cidade (1950-2008) [tesis de doctorado, Universidade Estadual Paulista]. Repositorio Institucional UNESP. https://repositorio.unesp.br/handle/11449/103123

Gomes, P. C. C. (1995). O conceito de região e sua discussão. En I.E.Castro (Org.), Geografia: conceitos e temas (pp. 49-73). Bertrand Brasil.

Gómez, E. M. (2007). Referentes históricos de la utilización de actividades en Sudamérica. En L. Paganizzi (Org.), Terapia ocupacional psicosocial (pp.23-30). Polemos.

Gómez Lillo, S. (2012). Antecedentes, creación y desarrollo de la terapia ocupacional en Chile: 50 años de historia. Abarca Girard Ediciones.

Gonzalez, L. (1959). Reunión inaugural. En Anales del Congreso Argentino De Rehabilitación del Lisiado (p.p. 19-26). Archivo Tesone.

Keohane, R. y Nye, J. (1977). Power and interdependence: world politics in transition. Little, Brown and Company.

Laval, E. R. (2007). Anotaciones para la historia de la poliomielitis en Chile. Revista Chilena de Infectología, 24(3), 247250. http://dx.doi.org/10.4067/S071610182007000300017

Le Goff, J. (2005). A história nova. Martins Fotes.
Llistar, D. B. (2009). Anticooperación: interferencia norte-sur: los problemas del sur global no se resuelven com más ayuda internacional. Icaria Antrazyt.

Lopes, R. E. (1991). A formação do terapeuta ocupacional: o currículo: histórico e propostas alternativas [tesis de maestría no publicada, Universidad Federal de São Carlos].

Lopes, R. E. (1997). A direção que construímos: algumas reflexões sobre a formação do terapeuta ocupacional. Revista de Terapia Ocupacional da USP, 4(7), 27-35.

Lopes, R. E. (2016). Cidadania, direitos e terapia ocupacional social. En R. E. Lopes y A. P. S. Malfitano (Org.), Terapia ocupacional social: desenhos teóricos e contornos práticos (pp. 29-48). EdUFSCar.

Lopes, R. E. y Hahn, M. S. (2004). The education of occupational therapists in Brazil: history and perspectives. WFOT Bulletin, 49(1), 24-32. https://doi.org/10.1179/ otb.2004.49.1.007

Macdonald, M. (1959). Conferencia la terapia ocupacional en la rehabilitación del Lisiado. En Anales del Congreso Argentino de Rehabilitación del Lisiado (p.p. 625-635). Archivo Tesone.

Machado, M. H., Oliveira, E. S. y Moyses, N. M. N. (2010). Tendências do mercado de trabalho em saúde no Brasil. En Anales de la Conferência Internacional Sobre Pesquisas em Recursos Humanos em Saúde. EAD/Ensp.

Magalhães, L. V. (1989). Os terapeutas ocupacionais no Brasil: sob o signo da contradição. [Tesis de Maestría, Universidade de Campinas]. 
Malagón, V. y Ávila, G. (1982). Estado actual de la poliomielitis en Colombia. Academia Nacional de Medicina.

Melo, D. O. C. V. (2015). Em busca de um ethos: narrativas da fundação da terapia ocupacional na cidade de São Paulo (1956-1969). [Tesis de Maestría, Universidade Federal de São Paulo].

Mendes, E. V. (1996). Uma agenda para a saúde. Hucitec.

Metaxas V. A. (2000). Eleanor Clarke Slagle and Susan E. Tracy: personal and professional identity and the development of occupational therapy in Progressive Era America. Nurs Hist Rev. (8) 39-70.

Milani, C. R. S. (2014). Evolução histórica da cooperação norte-sul. En Souza, A. M. (Org.), Repensando a cooperação internacional para o desenvolvimento (pp. 33-56). Ipea.

Millani, H. F. B. y Valente, M. L. L. C. (2008). O caminho da loucura e a transformação da assistência aos portadores de sofrimento mental. Revista Eletrônica Saúde Mental Álcool e Drogas, 4(2), 1-19.

Miranda, M. (2007). El compromiso con la ciencia. conocimiento y técnica en el trabajo social. Revista Internacional de Ciencias Sociales y Humanidades, (2), 9-28.

Morrison J. R., Olivares A. D., Graus R. J. M., Cifuentes L. D., Macari L. C. y Rojas P. E. (2016). Silvia Gómez Lillo y cincuenta años de terapia ocupacional en Chile: una biografía. TOG (A Coruña), 13(24), 1-27.

Morrison, R. J. (2014). La filosofía pragmatista en la terapia ocupacional de Eleanor Clarke Slagle: antecedentes epistemológicos e históricos desde los estudios feministas sobre la ciencia. [Tesis de Doctorado, Universidad de Salamanca].

Morrison, R. J. (2016). Los comienzos de la terapia ocupacional en Estados Unidos: una perspectiva feminista desde los estudios de Ciencia, Tecnología y Género (siglos XIX y XX). Historia Crítica, 62, 97-117.

Nabergoi, M., Mattei, M., Mulholland, M., Martínez, P. B., M., Spallato, N., Zorzoli, F., y García, A. S. (2013). Cooperación técnica en rehabilitación. Procesos iniciales de constitución de la formación académica y ejercicio profesional en Argentina. En $\mathrm{Li}$ bros de resúmenes del $1^{\circ}$ Congreso Chileno y $8^{\circ}$ Jornadas Nacionales de Terapia Ocupacional.

Nascimento, B. A. (1991). Loucura, trabalho e ordem: o uso do trabalho e da ocupação em instituições psiquiátricas. [Tesis de Maestría, Pontifícia Universidade Católica].

Nascimento, D. R. (Org.). (2010). A história da poliomielite. Garamond.

Papadópulos, J. y Radakovich, R. (2006). Educación Superior y género en América Latina y el Caribe. En IESALC-UNESCO, Informe sobre la Educación Superior en América Latina y el Caribe 2000-2005. La metamorfosis de la educación superior (pp.117128). ESALC.

Pollard, N., Sakellariou, D., y Kronenberg, F. (2009). A political practice of occupational therapy. Elsevier.

Queiroz, D. M. (2000). Mulheres no ensino superior no Brasil. En Anales de la Reunião Anual da Associação Nacional de Pesquisa e Pós-Graduação em Educação. (p.p. 1-23). 
Quiroga, V. A. M. (1995). Occupational therapy: the first thirty years, 1900-1930. American Occupational Therapy Association.

Reis, S. C. C. A. G. (2017). Histórias e memórias da institucionalização acadêmica da terapia ocupacional no Brasil: de meados da década de 1950 a 1983. [Tesis de Maestría, Universidade Federal de São Carlos].

Reis, S. C. C. A. G., y Lopes, R. E. (2018). O início da trajetória de institucionalização acadêmica da terapia ocupacional no Brasil: o que contam os(as) docentes pioneiros(as) sobre a criação dos primeiros cursos. Cadernos Brasileiros de Terapia Ocupacional, 26(2), 255-270. https://doi. org/10.4322/2526-8910.ctoAO1154

Rivas, A. P., Forn, C. Z., Muñoz, A. S., Matos, C., Castro, L., Pulido, S. y Sardi, T. (2013). Historia de la terapia ocupacional em Venezuela. En Anales del Congreso de la Confederación Latinoamericana de Terapeutas Ocupacionales, (p.p. 1-71).

Rodríguez Mendoza, L., Camargo Carrero, N., \& Escobar Jiménez, X. (2017). Terapia Ocupacional: una perspectiva histórica desde la Universidad Nacional de Colombia (1966-1989). Revista Ocupación Humana, 16(2), 26-45. https://doi. org/10.25214/25907816.132

Sanz-Valer, P., Rubio-Ortega, C., y Pastor-Montaño, M. A. (2013). La influencia de las ayudantes de reconstrucción en la historia de la terapia ocupacional. TOG (A Coruña), 18, 1-24.
Sbriller, L. (1997). Introducción a terapia ocupacional: marcos conceptuales. Catálogos.

Soares, L. B. T. (1991). Terapia ocupacional: lógica do capital ou do trabalho? Retrospectiva histórica da profissão no Estado brasileiro de 1950 a 1980 . Huitec.

Souza, A. (2011). América Latina, conceito e identidade: algumas reflexões da história. Revista de Humanidades do Curso de Ciências Sociais da UNIFAP, Macapá, 4(4), 29-39.

Testa, D. (2012). Aportes para el debate sobre los inicios de la profesionalización de la Terapia Ocupacional en Argentina. Revista Chilena de Terapia Ocupacional, 12 (1), 67-78.

Testa, D. y Spampinato, S. (2010). Género, salud mental y terapia ocupacional: algunas reflexiones sobre la influencia de la historia de las mujeres y la perspectiva de género en nuestras prácticas. Revista de Terapia Ocupacional de la Universidad de São Paulo, 21 (2), 174-181.

Trujillo, A. (2002). Terapia ocupacional: conocimiento y práctica en Colombia. Universidad Nacional. 